\title{
Classical dilute relativistic plasma in equilibrium. Two-particle distribution function
}

\author{
R. Lapiedra and E. Santos \\ Departamento de Física Teórica, Universidad de Santander, Santander, Spain
}

(Received 29 July 1981; revised manuscript received 27 July 1982)

\begin{abstract}
We consider here a dilute hot plasma which is homogeneous and in equilibrium in the framework of classical relativistic statistical mechanics as formulated recently by the authors. No external fields are present. The two-particle distribution function of the plasma is calculated for any temperature. This distribution will be physically significant, as long as the creation of pairs can be neglected. Several limits which apply at low or high temperatures are discussed. Comparison with previous work from several authors is made.
\end{abstract}

\section{INTRODUCTION}

This is the first in a set of two papers dealing with classical relativistic plasmas in equilibrium. We will start with a few dimensional considerations in order to fix the limits of validity of the calculational framework used in these papers.

We consider a system of particles which may have different charges and masses. For dimensional purposes it is convenient to consider the typical charge $e$ and the smallest mass $m$. In practice, $m$ and $e$ will be the electron mass and charge. The combination of these two parameters with the universal constants $\hbar$ (Planck constant) and $c$ (speed of light) makes it possible to obtain a single dimensionless number $\alpha \equiv\left(e^{2} / \hbar c\right)$ which, for the elementary charge, takes the well-known value $\alpha=\frac{1}{137}$. A homogeneous plasma is characterized by two parameters: the density of particles $\rho$ and the temperature $T$ (if there are several kinds of particles it is also necessary to state their concentrations). There are different energies associated with the plasma, namely, (1) energy of the rest mass per particle $m c^{2}$, (2) kinetic energy per particle of order $k T, k$ being the Boltzmann constant, and (3) Coulomb energy of order $e^{2} \rho^{1 / 3}$ per particle.

The ratios of these energies give us the two main dimensionless parameters of the plasma:

$$
\epsilon \equiv k T / m c^{2}, \epsilon_{d} \equiv e^{2} \rho^{1 / 3} / k T .
$$

In what follows we study the ranges of $\epsilon$ and $\epsilon_{d}$ for which our calculations are valid.

The first question is whether the plasma can be considered classical. The system is safely classical if $\hbar \equiv \hbar / p<<\rho^{-1 / 3}, p$ being the typical linear momentum of the particles. This leads, for not very relativistic plasmas, to the condition

$$
\epsilon_{d} \ll \alpha \epsilon^{-1 / 2} \sim 10^{-2} \epsilon^{-1 / 2} \lesssim 10^{-2}
$$

Nevertheless, we must take into account that, strictly speaking, a classical system of charged particles cannot be in equilibrium because the energy is not bounded from below (the Coulomb energy of a pair of particles with opposite charges can be indefinitely negative). Therefore, we must resort to the quantum behavior to prevent collapse. In consequence, all classical predictions about correlations at very short distances will be meaningless.

Another important point is that of the walls. We shall study a plasma which is homogeneous and isotropic but the plasma must be confined or it will expand. We may assume that the plasma is confined by some kind of walls which prevent the escape of particles, but that the container is so large that the effects of the walls are negligible.

The starting point of our treatment is the "predictive relativistic mechanics" (PRM). ${ }^{1}$ As this theory is not widely known, we present its main idea as follows. Assume two isolated particles which, in a given frame, have positions and velocities $\vec{r}_{1}(0), \vec{v}_{1}$, and $\overrightarrow{\mathbf{r}}_{2}(0), \overrightarrow{\mathbf{v}}_{2}$, respectively, at time $t=0$. If the particles are noninteracting, their paths are

$$
\overrightarrow{\mathrm{r}}_{j}(t)=\overrightarrow{\mathrm{r}}_{j}(0)+\overrightarrow{\mathrm{v}}_{j} t, j=1,2
$$

even if the velocities are close to that of light. If the particles are charged, then Eqs. (3) do not give the actual paths, but if the charge $e$ of each particle is very small then Eqs. (3) are approximately correct for $t$ not too large. A correction of order $e^{2}$ to the paths (3) can be obtained by calculating from Eqs. (3) the (retarded) electromagnetic force of particle $1(2)$ on particle 2(1) and solving afterwards the (relativistic) equations of motion. The procedure can be repeated and, finally, the correct paths of the particles can be expressed as an expansion in powers of $e$ Eqs. (3) being the zeroth-order term in that expansion. Predictive relativistic mechanics is a theory that can be seen as the rigorization and generaliza- 
tion of this idea. Its main achievement is the possibility of writing the equations of motion of a system of $N$ interacting relativistic particles as a set of $N$ (Newtonian-like) second-order differential equations. The fields do not appear explicitly in the formalism, but one may obtain equations which lead to exactly the same results as conventional electrodynamics. The formalism allows the calculation of the energy, the momentum, and the angular momentum of the system of $N$ particles as functions of the positions and velocities of these particles. These quantities include the contributions to the energy, momentum, and angular momentum usually associated with the fields. In practice, the differential equations of PRM can be written only up to a given order in powers of the charges. The expression up to second order in the charges has been evaluated explicitly ${ }^{2}$ and it will be our starting point [see Eq. (16) below]. Note that no expansion in $v / c$ is involved.

In a plasma an expansion in powers of the typical charges is actually an expansion in powers of the parameter $\epsilon_{d}$ introduced in (1), which is the "dilution parameter." Therefore, our study is only valid for dilute plasmas $\left(\epsilon_{d}<<1\right)$. This is convenient because a similar inequality for $\epsilon_{d}$ is obtained from the following consideration. The method that we use for the study of the thermodynamics of the plasma is a generalization to relativistic systems of the Bogoliubov method which starts from the BogoliubovBorn-Green-Kirkwood-Yvon (BBGKY) hierarchy cutoff at the level of the two-body distribution functions. In fact, this method provides a first-order approximation in the density parameter $\epsilon_{d}$. On the other hand, we do not set, in principle, a limit on the value of the parameter $\epsilon$ of (1) which measures the relativistic character of the plasma. Actually, when $\epsilon$ is greater than or of the same order as 1, the creation of electron-positron pairs becomes possible and our classical treatment loses all its meaning.

A final point which deserves attention is that of the radiation. As we have stated above, PRM takes into account all interactions between relativistic particles, including the radiation produced by the particles themselves. ${ }^{3,4}$ Radiation is an effect of order $e^{6}$ and, therefore, is not included in our treatment. Nevertheless, radiation may be important because, even a small rate of radiation produces a cooling of the plasma which may be large after a long time. Therefore, a more carful analysis is worthwhile.

If we want to study a plasma strictly in equilibrium (i.e., it does not change in an indefinitely large time interval), then we must assume that the walls of the container are perfectly reflecting for the radiation. Then, the radiation comes to the plasma after reflected by the walls and this effect is not taken into account by PRM. We need to assume that, aside from the interacting particles, we have a background of (external) radiation which interacts with them. An estimation of the relevance of that radiation is obtained if we assume that is is a blackbody radiation with Planck spectrum, whose energy density is given by Stephan's law. If we calculate the ratio $r$ of that energy to the typical Coulomb energy of neighboring particles in the plasma we obtain

$$
r=\left(\pi^{2} / 60\right)\left(e^{2} / \hbar c\right)^{3} \epsilon_{d}^{-4} .
$$

If this ratio is much smaller than one, we may neglect the effects of the radiation. This gives the condition

$$
\epsilon_{d} \gg\left(e^{2} / \hbar c\right)^{3 / 4} \simeq 10^{-2} .
$$

In the opposite direction, if $\epsilon_{d}$ is very small (much less than 0.01) a good approximation should be to assume that each particle interacts with the radiation but not with other particles (except through radiation). Then the plasma can be studied considering a single particle in a random background radiation, which leads to the relativistic Boltzmann distribution. ${ }^{5}$

A comparison of (2) and (5) shows that a plasma strictly in equilibrium can be studied with our formalism only if $\epsilon \ll 1$, i.e., the plasma can be only slightly relativistic.

Actual plasmas do fulfill (2) but do not fulfill (5). For instance, fusion plasmas with magnetic confinement have $\epsilon_{d} \simeq 10^{-5}$ and $\epsilon \simeq 10^{-2}$. Nevertheless, our framework can be applied to these plasmas as follows. In a fusion plasma radiation escapes through the walls so that there is no equilibrium, strictly speaking. However, the loss of energy by radiation is an extremely slow process in a dilute plasma. A characteristic time for the process is the ratio between the energy per particle of order $k T$, and the power emitted. A particle of the plasma has a typical acceleration of order $e^{2} \rho^{2 / 3} / m$ so that the radiated power per particle is of order $e^{6} \rho^{4 / 3} m^{-2} c^{-3}$. Therefore, the cooling time should be of order

$$
t=\epsilon^{-2} \epsilon_{d}^{-3} \rho^{-1 / 3} c^{-1}
$$

which for fusion plasmas is of the order of $10^{3} \mathrm{sec}$. This macroscopic time is presumably much larger than any relaxation time in a near-equilibrium plasma. This enables us to consider our radiating plasma as nearly in equilibrium at a given time.

\section{GENERAL FRAMEWORK: RELATIVISTIC BBGKY HIERARCHY AND TWO-PARTICLE CORRELATIONS}

In this paper we calculate the classical twoparticle distribution function of a dilute relativistic plasma. No external fields are present and so the 
plasma is homogeneous and isotropic. In principle, the calculation is valid for any temperature $T$. However, as has been pointed out in Sec. I, for temperatures such that $\epsilon \equiv k T / m c^{2} \geq 1$ the creation of pairs becomes possible and our classical treatment loses its meaning. In a future paper this twoparticle distribution function will be used to work out the thermodynamics of the plasma to first order in $\epsilon$.

Our calculations are grounded in the classical relativistic statistical mechanics developed by the authors $^{6}$ in the framework of predictive relativistic mechanics. Before summarizing here the prominent facts of this formulation of the statistical mechanics, let us state the basic notation of this paper: For a macroscopic system of $N$ particles, $x_{a}^{\alpha}$ stands for the four-position of the particle $\underline{a}$, which means that $a, b, \ldots=1, \ldots, N$ and $\alpha, \beta, \ldots=0,1,2,3$ and in an obvious notation $x_{a}^{\alpha}=\left(t_{a}, \overrightarrow{\mathbf{x}}_{a}\right)$. Also, $\overrightarrow{\mathbf{u}}_{a}$ stands for the space components of the four-velocity $u_{a}^{\alpha}$ of the particle $\underline{a}$ that is, $\overrightarrow{\mathbf{u}}_{a}=\gamma_{a} \overrightarrow{\mathbf{v}}_{a}$, with $\gamma_{a}=\left(1-v_{a}^{2}\right)^{1 / 2}$ and $\overrightarrow{\mathbf{v}}_{a}$ the three-velocity. The speed of light $c$ is taken equal to one. Then, in Ref. 6, we define the generalized distribution function

$$
F\left(t_{1}, \overrightarrow{\mathbf{x}}_{1}, \overrightarrow{\mathrm{u}}_{1}, \ldots, t_{N}, \overrightarrow{\mathrm{x}}_{N}, \overrightarrow{\mathrm{u}}_{N}\right) \equiv F\left(t_{a}, \overrightarrow{\mathrm{x}}_{a}, \overrightarrow{\mathrm{u}}_{a}\right),
$$

where the word "generalized" refers to the fact that in this distribution function we have, in principle, a different time for every particle. Now if we take $t_{a}=t, \forall a$, we recover the usual distribution function $f\left(t, \overrightarrow{\mathrm{x}}_{a}, \overrightarrow{\mathrm{u}}_{a}\right)$, that is,

$$
f\left(t, \overrightarrow{\mathrm{x}}_{a}, \overrightarrow{\mathrm{u}}_{a}\right)=F\left(t_{a}=t, \overrightarrow{\mathrm{x}}_{a}, \overrightarrow{\mathrm{u}}_{a}\right) .
$$

For the generalized distribution function $F$ there are, in our formalism, $N$ continuity equations which constitute a system of differential equations for $F$, which are compatible as a consequence of the fundamental equations of predictive relativistic mechanics. $^{6}$

Then we consider in Ref. 6 the case of a relativistic dilute plasma which is isolated (i.e., no external fields). There, and here, we assume that the dilution parameter $\epsilon_{d}$ is small enough. The zeroth order in $\epsilon_{d}$ corresponds to the ideal gas and in Ref. 6 and in this paper, we are interested in calculating the lowest corrections to that. According to what has been explained there, to lowest order in the charges, i.e., $e^{2}$, only two-body interactions need to be considered. Therefore, the acceleration produced on a given charge by the electromagnetic field created by all others is a sum of terms, each one due to one particle. In this way, the problem of the interactions in the plasma reduces to the calculation of the acceleration produced on one particle, say 1 , by another one, say $\underline{2}$. Now, this problem can be dealt with as if only particles $\underline{1}$ and $\underline{2}$ exist in the universe, all corrections to that model being of higher order in $e$. At first sight, the calculation of the relativistic interaction between two charged particles seems rather involved, because the force on particle 1 at time $t$ would depend on the position and velocity of particle $\underline{2}$ at a retarded time. Also, the position and velocity of 1 at that time depends on the position and velocity of 1 at an earlier time, and so on. As we have said in Sec. I, the main success of predictive relativistic mechanics has been to show that the selfconsistent motion of two interacting particles 1 and $\underline{2}$ can be solved in a relatively easy way be writting a pair of coupled Newton-like (differential secondorder) equations whose solutions give all the compatible paths for the pair of particles. If one chooses a frame of reference, the acceleration on particle 1 due to the presence of $\underline{2}$ can be calculated from the position and velocity of $\underline{2}$ at that time. This does not mean that actions propagate instantaneously, but rather that there is a precise scheme to take retardation into account automatically, through the equations of the theory. All we shall need in that paper is the time component of the acceleration of particle $\underline{2}$ due to particle 1 , which has been calculated to second order in the charges in Ref. 2. Its actual expression can be seen below [see Eq. (16)].

The fact that the basic interaction in a dilute plasma splits into two-body interactions allows us to reduce the $N$ continuity equations for the generalized distribution $F$ to $N$ Liouville equations and hence we obtain ${ }^{6}$ the relativistic BBGKY hierarchy

$$
\begin{aligned}
u_{A}^{\alpha} \frac{\partial F^{(s)}}{\partial x_{A}^{\alpha}} & +\sum_{B} \xi_{A B}^{\alpha} \frac{\partial F^{(s)}}{\partial u_{A}^{\alpha}} \\
& +\sum_{R} \int \xi_{A R}^{\alpha} \frac{\partial F^{(s+1)}}{\partial u_{A}^{\alpha}} d^{3} x_{R} d^{3} u_{R}=0, \quad B \neq A
\end{aligned}
$$

where $F^{(s)}$ is the reduced generalized distribution function

$$
F^{(s)}\left(t_{A}, \overrightarrow{\mathrm{x}}_{A}, \overrightarrow{\mathrm{u}}_{A}\right)=\int F \prod_{R=s+1}^{N} d^{3} x_{R} d^{3} u_{R} .
$$

Here $s=1,2, \ldots, N-1$. The first capital letters $A, B, \ldots$ run from 1 to $s$ and the latest ones $R, S, \ldots$ from $s+1$ to $N$. Finally, $\xi_{A B}^{\alpha}$ is the acceleration of the charge $A$ in the presence of the charge $B$ to first order in the product of the charges, i.e., $\xi_{A}^{\alpha}=\sum_{a} \xi_{A a}^{\alpha}+i s$, to this order, the acceleration of the charge $A$, since as mentioned before, in this approximation we only have two-body interactions.

In (7) the Einstein summation convention is only 
valid for Greek labels. Therefore, for each value of $s$ we have $s$ equations, since $A=1,2, \ldots . s$. As in the nonrelativistic case, the determination of the reduced generalized distribution function $F^{(s)}$ can only be made when the hierarchy is cut off somewhere, that is, when for some value $s$ we give $F^{(s+1)}$ as a function of the other $F^{(r)}$ functions with $r<s+1$. According to this we have, for any particles $\underline{1}, \underline{2}, \underline{3}$,

$$
\begin{aligned}
& F^{(2)}(1,2)=F^{(1)}(1) F^{(1)}(2)[1+g(1,2)], \\
& F^{(3)}(1,2,3)=F^{(1)}(1) F^{(1)}(2) F^{(1)}(3)[1+g(1,2)+g(1,3)+g(2,3)],
\end{aligned}
$$

in analogy with the nonrelativistic case. ${ }^{7}$ It is supposed that the "correlation function" $g(1,2)$ is much smaller than 1.

Let us now treat the more particular case of when the dilute plasma is homogeneous and in equilibrium. Then, putting (9) and (10) into (7) for $s=1,2$, we obtain

$$
\begin{aligned}
& \sum_{R} \int F^{(1)}(R) \xi_{1 R}^{\alpha} \partial\left[F^{(1)}(1) g(1, R)\right] / \partial u_{1}^{\alpha} d^{3} x_{R} d^{3} u_{R}=0, \\
& u_{A}^{\alpha} \frac{\partial g(1,2)}{\partial x_{A}^{\alpha}}=\beta^{\prime} m_{A} \xi_{A B}^{0}+\beta^{\prime} m_{A} \sum_{R} \int F^{(1)}(R) \xi_{A R}^{0} g(B, R) d^{3} x_{R} d^{3} u_{R} .
\end{aligned}
$$

Here, for the one-particle distribution function $F^{(1)}$ we have the relativistic Maxwellian distribution

$F^{(1)}(1)=\left[\beta^{\prime} m_{1} \exp \left(-\beta^{\prime} m_{1} \gamma_{1}\right)\right] / 4 \pi V K_{2}\left(\beta^{\prime} m_{1}\right)$,

where $m_{1}$ is the mass of the particle labeled $\underline{1}, V$ is the volume of the system, and $K_{2}$ is the modified second-order Bessel function. Equation (13) is the free relativistic Maxwellian distribution except for the constant $\beta^{\prime}$ which replaces here the Boltzmann factor $\beta \equiv 1 / k T$. According to Kosachev and Trubnikov, ${ }^{8} \beta^{\prime}$ coincides with $\beta$ in the nonrelativistic limit, but in general we have $\beta^{\prime} \neq \beta$ because of the relativistic interaction terms. We shall discuss this point further in a later paper.

Then, in order to get the two-particle generalized distribution function $F^{(2)}(1,2)$ of the plasma, we must solve the integro-differential equation (12) with the supplementary condition (11). In this work we will be concerned only with the usual two-particle distribution function $f^{(2)}(1,2)=\left.F^{(2)}(1,2)\right|_{t_{1}=t_{2}}$.
Next, Sec. III is devoted to the explicit calculation of $f^{(2)}(1,2)$ starting from Eq. (12) and condition (11), and in Sec. IV we consider the special case of a slightly relativistic plasma and we compare our results with previous ones.

\section{TWO-PARTICLE DISTRIBUTION FUNCTION FOR A RELATIVISTIC DILUTE AND HOMOGENEOUS PLASMA IN EQUILIBRIUM WITHOUT EXTERNAL FIELDS}

The calculations of this section are valid, in principle, whatever the temperature of the plasma but, in fact, for a temperature of the order of the equivalent temperature $T_{e}$ of electrons $\left(k T_{e}\right.$ equal rest mass of the electron) the creation of electronpositron pairs should be considered, which is not done here.

From Eqs. (1) with $A=1,2$, respectively, and setting $t_{1}=t_{2}=t_{R}, \forall R$, we obtain (see Ref. 6)

$$
\begin{array}{r}
\overrightarrow{\mathrm{v}}_{1} \cdot \frac{\partial G(1,2)}{\partial \overrightarrow{\mathrm{x}}_{1}}-\left.\beta^{\prime} m_{1} \gamma_{1}^{-1} \xi_{12}^{0}\right|_{0}-\left.\beta^{\prime} m_{1} \gamma_{1}^{-1} \sum_{R} \int \xi_{1 R}^{0}\right|_{0} F^{(1)}(R) G(2, R) d^{3} x_{R} d^{3} u_{R}+\overrightarrow{\mathrm{v}}_{2} \cdot \frac{\partial G(1,2)}{\partial \overrightarrow{\mathrm{x}}_{2}} \\
-\left.\beta^{\prime} m_{2} \gamma_{2}^{-1} \xi_{21}^{0}\right|_{0}-\left.\beta^{\prime} m_{2} \gamma_{2}^{-1} \sum_{R} \int \xi_{2 R}^{0}\right|_{0} F^{(1)}(R) G(1, R) d^{3} x_{R} d^{3} u_{R}=0
\end{array}
$$

where the notation

$$
\left.G(1,2) \equiv G\left(\vec{x}, \vec{v}_{1}, \vec{v}_{2}\right) \equiv g(1,2)\right|_{t_{1}=t_{2}},\left.\left.\quad \xi_{12}^{0}\right|_{0} \equiv \xi_{12}^{0}\right|_{t_{1}=t_{2}}, \quad \vec{x} \equiv \vec{x}_{1}-\vec{x}_{2}
$$

has been used. Notice that only the time components $\xi_{12}^{0}$ and $\xi_{21}^{0}$ of the four vectors $\xi_{12}^{\alpha}, \xi_{21}^{\alpha}$ appear in Eq. (14).

For $\left.\xi_{12}^{0}\right|_{0}$ we have the expression ${ }^{2}$

$$
\left.\xi_{12}^{0}\right|_{0}=e_{1} e_{2} \gamma_{1} \gamma_{2} \overrightarrow{\mathrm{x}} \cdot \overrightarrow{\mathrm{v}}_{1} / m_{1} R_{12}^{3} \text {, }
$$


where $R_{12} \equiv\left[r^{2}+\left(\overrightarrow{\mathrm{x}} \cdot \overrightarrow{\mathrm{u}}_{2}\right)^{2}\right]^{1 / 2}, r^{2} \equiv \overrightarrow{\mathrm{x}}^{2}$ and $e_{1}, e_{2}$ are the charges of particles $\underline{1}$ and $\underline{2}$. Then Eq. (14) becomes $\overrightarrow{\mathrm{v}}_{1} \cdot\left(\frac{\partial G(1,2)}{\partial \overrightarrow{\mathrm{x}}_{1}}-\beta^{\prime} m_{1} \sum_{R} \int \vec{\zeta}_{1 R} F^{(1)}(R) G(R, 2) d^{3} x_{R} d^{3} u_{R}-\beta^{\prime} m_{1} \vec{\xi}_{12}\right)$

$$
+\overrightarrow{\mathbf{v}}_{2} \cdot\left(\frac{\partial G(1,2)}{\partial \overrightarrow{\mathbf{x}}_{2}}-\beta^{\prime} m_{2} \sum_{R} \int \vec{\xi}_{2 R} F^{(1)}(R) G(R, 1) d^{3} x_{R} d^{3} u_{R}-\beta^{\prime} m_{2} \vec{\xi}_{21}\right)=0
$$

with

$$
\vec{\zeta}_{12}=e_{1} e_{2} \gamma_{2} \overrightarrow{\mathrm{x}} / m_{1} R_{12}^{3}
$$

for any pair of particles $\underline{1}$ and $\underline{2}$.

The Fourier transform of Eq. (17) gives

$$
\begin{array}{r}
G\left(\overrightarrow{\mathrm{k}}, \overrightarrow{\mathrm{v}}_{1}, \overrightarrow{\mathrm{v}}_{2}\right) \overrightarrow{\mathrm{k}} \cdot \overrightarrow{\mathrm{v}}_{1}+(2 / \pi)^{1 / 2} \beta^{\prime} e_{1} e_{2} \frac{\overrightarrow{\mathrm{k}} \cdot \overrightarrow{\mathrm{v}}_{1}-\left(\overrightarrow{\mathrm{k}} \cdot \overrightarrow{\mathrm{v}}_{2}\right)\left(\overrightarrow{\mathrm{v}}_{1} \cdot \overrightarrow{\mathrm{v}}_{2}\right)}{k^{2}-\left(\overrightarrow{\mathrm{k}} \cdot \overrightarrow{\mathrm{v}}_{2}\right)^{2}} \\
+4 \pi \beta^{\prime} e_{1} \sum_{R} e_{R} \int \frac{\overrightarrow{\mathrm{k}} \cdot \overrightarrow{\mathrm{v}}_{1}-\left(\overrightarrow{\mathrm{k}} \cdot \overrightarrow{\mathrm{v}}_{R}\right)\left(\overrightarrow{\mathrm{v}}_{1} \cdot \overrightarrow{\mathrm{v}}_{R}\right)}{k^{2}-\left(\overrightarrow{\mathrm{k}} \cdot \overrightarrow{\mathrm{v}}_{R}\right)^{2}} F^{(1)}(R) G\left(\overrightarrow{\mathrm{k}}, \overrightarrow{\mathrm{v}}_{2}, \overrightarrow{\mathrm{v}}_{R}\right) d^{3} u_{R}-G\left(\overrightarrow{\mathrm{k}}, \overrightarrow{\mathrm{v}}_{1}, \overrightarrow{\mathrm{v}}_{2}\right) \overrightarrow{\mathrm{k}} \cdot \overrightarrow{\mathrm{v}}_{2} \\
-4 \pi \beta^{\prime} e_{2} \sum_{R} e_{R} \int \frac{\overrightarrow{\mathrm{k}} \cdot \overrightarrow{\mathrm{v}}_{2}-\left(\overrightarrow{\mathrm{k}} \cdot \overrightarrow{\mathrm{v}}_{R}\right)\left(\overrightarrow{\mathrm{v}}_{2} \cdot \overrightarrow{\mathrm{v}}_{R}\right)}{k^{2}-\left(\overrightarrow{\mathrm{k}} \cdot \overrightarrow{\mathrm{v}}_{R}\right)^{2}} F^{(1)}(R) G\left(\overrightarrow{\mathrm{k}}, \overrightarrow{\mathrm{v}}_{1}, \overrightarrow{\mathrm{v}}_{R}\right) d^{3} u_{R}=0, \quad(19) \\
k^{2}-\left(\overrightarrow{\mathrm{k}} \cdot \overrightarrow{\mathrm{v}}_{1}\right)^{2}
\end{array}
$$

where $G\left(\overrightarrow{\mathrm{k}}, \overrightarrow{\mathrm{v}}_{1}, \overrightarrow{\mathrm{v}}_{2}\right)$ is the Fourier transform of $G(1,2) \equiv G\left(\overrightarrow{\mathrm{x}}, \overrightarrow{\mathrm{v}}_{1}, \overrightarrow{\mathrm{v}}_{2}\right)$, i.e.,

$$
G\left(\overrightarrow{\mathrm{k}}, \overrightarrow{\mathrm{v}}_{1}, \overrightarrow{\mathrm{v}}_{2}\right)=(2 \pi)^{-3 / 2} \int G\left(\overrightarrow{\mathrm{x}}, \overrightarrow{\mathrm{v}}_{1}, \overrightarrow{\mathrm{v}}_{2}\right) \exp (i \overrightarrow{\mathrm{k}} \cdot \overrightarrow{\mathrm{x}}) d^{3} x \text {. }
$$

The linearity of Eq. (19) and the functional form of the inhomogeneous term suggest trying the following "ansatz":

$$
G\left(\overrightarrow{\mathrm{k}}, \overrightarrow{\mathrm{v}}_{1}, \overrightarrow{\mathrm{v}}_{2}\right)=e_{1} e_{2} \sum_{i=1}^{5} A_{i}(k) G\left(\overrightarrow{\mathrm{n}}, \overrightarrow{\mathrm{v}}_{1}, \overrightarrow{\mathrm{v}}_{2}\right), \quad \overrightarrow{\mathrm{n}} \equiv \overrightarrow{\mathrm{k}} / k
$$

with

$$
\begin{aligned}
& G_{1}=1, \quad G_{2}=\frac{\left(\overrightarrow{\mathrm{n}} \cdot \overrightarrow{\mathrm{v}}_{1}\right)\left(\overrightarrow{\mathrm{n}} \cdot \overrightarrow{\mathrm{v}}_{2}\right)}{\left[1-\left(\overrightarrow{\mathrm{n}} \cdot \overrightarrow{\mathrm{v}}_{1}\right)^{2}\right]\left[1-\left(\overrightarrow{\mathrm{n}} \cdot \overrightarrow{\mathrm{v}}_{2}\right)^{2}\right]}, \quad G_{3}=\frac{\left(\overrightarrow{\mathrm{n}} \cdot \overrightarrow{\mathrm{v}}_{1}\right)^{2}\left(\overrightarrow{\mathrm{n}} \cdot \overrightarrow{\mathrm{v}}_{2}\right)^{2}}{\left[1-\left(\overrightarrow{\mathrm{n}} \cdot \overrightarrow{\mathrm{v}}_{1}\right)^{2}\right]\left[1-\left(\overrightarrow{\mathrm{n}} \cdot \overrightarrow{\mathrm{v}}_{2}\right)^{2}\right]}, \\
& G_{4}=\frac{\overrightarrow{\mathrm{v}}_{1} \cdot \overrightarrow{\mathrm{v}}_{2}}{\left[1-\left(\overrightarrow{\mathrm{n}} \cdot \overrightarrow{\mathrm{v}}_{1}\right)^{2}\right]\left[1-\left(\overrightarrow{\mathrm{n}} \cdot \overrightarrow{\mathrm{v}}_{2}\right)^{2}\right]}, \quad G_{5}=\frac{\left(\overrightarrow{\mathrm{v}}_{1} \cdot \overrightarrow{\mathrm{v}}_{2}\right)\left(\overrightarrow{\mathrm{n}} \cdot \overrightarrow{\mathrm{v}}_{1}\right)\left(\overrightarrow{\mathrm{n}} \cdot \overrightarrow{\mathrm{v}}_{2}\right)}{\left[1-\left(\overrightarrow{\mathrm{n}} \cdot \overrightarrow{\mathrm{v}}_{1}\right)^{2}\right]\left[1-\left(\overrightarrow{\mathrm{n}} \cdot \overrightarrow{\mathrm{v}}_{2}\right)^{2}\right]} .
\end{aligned}
$$

Putting (20) into Eq. (19) we obtain

$$
\begin{aligned}
\sum_{i=1}^{5} A_{i}(k) G_{i}\left(\overrightarrow{\mathrm{n}}, \overrightarrow{\mathrm{v}}_{1}, \overrightarrow{\mathrm{v}}_{2}\right)+(2 / \pi)^{1 / 2} \frac{\beta^{\prime}}{k}\left[\frac{\overrightarrow{\mathrm{n}} \cdot \overrightarrow{\mathrm{v}}_{1}-\left(\overrightarrow{\mathrm{n}} \cdot \overrightarrow{\mathrm{v}}_{2}\right)\left(\overrightarrow{\mathrm{v}}_{1} \cdot \overrightarrow{\mathrm{v}}_{2}\right)}{1-\left(\overrightarrow{\mathrm{n}} \cdot \overrightarrow{\mathrm{v}}_{2}\right)^{2}}+\frac{\left(\overrightarrow{\mathrm{n}} \cdot \overrightarrow{\mathrm{v}}_{1}\right)\left(\overrightarrow{\mathrm{v}}_{1} \cdot \overrightarrow{\mathrm{v}}_{2}\right)-\overrightarrow{\mathrm{n}} \cdot \overrightarrow{\mathrm{v}}_{2}}{1-\left(\overrightarrow{\mathrm{n}} \cdot \overrightarrow{\mathrm{v}}_{1}\right)^{2}}\right] \\
+4 \pi \beta^{\prime} \sum_{R, i} e_{R}^{2} A_{i}(k) I_{i}\left(\beta^{\prime} m_{R}\right)=0,
\end{aligned}
$$

where $\overrightarrow{\mathbf{v}} \equiv \overrightarrow{\mathbf{v}}_{1}-\overrightarrow{\mathbf{v}}_{2}$ and where

$$
\begin{aligned}
I_{i}\left(\beta^{\prime} m_{R}\right) \equiv & \int F^{(1)}(R) G_{i}\left(\overrightarrow{\mathrm{n}}, \overrightarrow{\mathrm{v}}_{2}, \overrightarrow{\mathrm{v}}_{R}\right) \frac{\overrightarrow{\mathrm{k}} \cdot \overrightarrow{\mathrm{v}}_{1}-\left(\overrightarrow{\mathrm{k}} \cdot \overrightarrow{\mathrm{v}}_{R}\right)\left(\overrightarrow{\mathrm{v}}_{1} \cdot \overrightarrow{\mathrm{v}}_{R}\right)}{k^{2}-\left(\overrightarrow{\mathrm{k}} \cdot \overrightarrow{\mathrm{v}}_{R}\right)^{2}} d^{3} u_{R} \\
& -\int F^{(1)}(R) G_{i}\left(\overrightarrow{\mathrm{n}}, \overrightarrow{\mathrm{v}}_{1}, \overrightarrow{\mathrm{v}}_{R}\right) \frac{\overrightarrow{\mathrm{k}} \cdot \overrightarrow{\mathrm{v}}_{2}-\left(\overrightarrow{\mathrm{k}} \cdot \overrightarrow{\mathrm{v}}_{R}\right)\left(\overrightarrow{\mathrm{v}}_{2} \cdot \overrightarrow{\mathrm{v}}_{R}\right)}{k^{2}-\left(\overrightarrow{\mathrm{k}} \cdot \overrightarrow{\mathrm{v}}_{R}\right)^{2}} d^{3} u_{R}
\end{aligned}
$$

After some calculation we obtain 


$$
\begin{aligned}
& I_{1}\left(\beta^{\prime} m_{R}\right)=\frac{\overrightarrow{\mathrm{n}} \cdot \overrightarrow{\mathrm{v}}}{k V}, I_{2}\left(\beta^{\prime} m_{R}\right)=0, \\
& I_{3}\left(\beta^{\prime} m_{R}\right)=C\left(\beta^{\prime} m_{R}\right) \frac{\left(\overrightarrow{\mathrm{n}} \cdot \overrightarrow{\mathrm{v}}_{1}\right)\left(\overrightarrow{\mathrm{n}} \cdot \overrightarrow{\mathrm{v}}_{2}\right)(\overrightarrow{\mathrm{n}} \cdot \overrightarrow{\mathrm{v}})\left[1+\left(\overrightarrow{\mathrm{n}} \cdot \overrightarrow{\mathrm{v}}_{1}\right)\left(\overrightarrow{\mathrm{n}} \cdot \overrightarrow{\mathrm{v}}_{2}\right)\right]}{k\left[1-\left(\overrightarrow{\mathrm{n}} \cdot \overrightarrow{\mathrm{v}}_{1}\right)^{2}\right]\left[1-\left(\overrightarrow{\mathrm{n}} \cdot \overrightarrow{\mathrm{v}}_{2}\right)^{2}\right]}, I_{4}\left(\beta^{\prime} m_{R}\right)=0, \\
& I_{5}\left(\beta^{\prime} m_{R}\right)=\frac{1}{k} \frac{\overrightarrow{\mathrm{n}} \cdot \overrightarrow{\mathrm{v}}\left[1+\left(\overrightarrow{\mathrm{n}} \cdot \overrightarrow{\mathrm{v}}_{1}\right)\left(\overrightarrow{\mathrm{n}} \cdot \overrightarrow{\mathrm{v}}_{2}\right)\right]}{\left[1-\left(\overrightarrow{\mathrm{n}} \cdot \overrightarrow{\mathrm{v}}_{1}\right)^{2}\right]\left[1-\left(\overrightarrow{\mathrm{n}} \cdot \overrightarrow{\mathrm{v}}_{2}\right)^{2}\right]}\left\{\left[C\left(\beta^{\prime} m_{R}\right)-B\left(\beta^{\prime} m_{R}\right)\right]\left(\overrightarrow{\mathrm{n}} \cdot \overrightarrow{\mathrm{v}}_{1}\right)\left(\overrightarrow{\mathrm{n}} \cdot \overrightarrow{\mathrm{v}}_{2}\right)+B\left(\beta^{\prime} m_{R}\right) \overrightarrow{\mathrm{v}}_{1} \cdot \overrightarrow{\mathrm{v}}_{2}\right\}
\end{aligned}
$$

with $C\left(\beta^{\prime} m_{R}\right)$ and $B\left(\beta^{\prime} m_{R}\right)$ standing for the integrals

$$
\begin{aligned}
& C\left(\beta^{\prime} m_{R}\right)=V^{-1}+2 \pi \int \frac{F^{(1)}(R)}{V_{R}}\left(\ln \frac{1+v_{R}}{1-v_{R}}\right) u_{R}^{2} d u_{R}, \\
& B\left(\beta^{\prime} m_{R}\right)=\pi \int F^{(1)}(R)\left(\frac{3-v_{R}^{2}}{2 v_{R}} \ln \frac{1+v_{R}}{1-v_{R}}-3\right) u_{R}^{2} d u_{R} .
\end{aligned}
$$

Substitution of (25) into (23) gives, after a little algebra,

$$
A_{1}=\frac{(2 / \pi)^{1 / 2} \beta^{\prime}}{k^{2}+4 \pi \beta^{\prime} V^{-1} \sum_{R} e_{R}^{2}}, A_{2}=A_{3}=-A_{5}=\frac{(2 / \pi)^{1 / 2} \beta^{\prime}}{k^{2}+4 \pi \beta^{\prime} \sum_{R} e_{R}^{2} B\left(\beta^{\prime} m_{R}\right)}
$$

which according to (21) gives for $G\left(\vec{k}, \vec{v}_{1}, \vec{v}_{2}\right)$,

$$
\begin{aligned}
G\left(\overrightarrow{\mathrm{k}}, \overrightarrow{\mathrm{v}}_{1}, \overrightarrow{\mathrm{v}}_{2}\right)= & -\frac{(2 / \pi)^{1 / 2} \beta^{\prime} e_{1} e_{2}}{k^{2}+\kappa^{2}} \\
& +\frac{(2 / \pi)^{1 / 2} \beta^{\prime} e_{1} e_{2}}{k^{2}+\alpha^{2}} \frac{\left[1+\left(\overrightarrow{\mathrm{n}} \cdot \overrightarrow{\mathrm{v}}_{1}\right)\left(\overrightarrow{\mathrm{n}} \cdot \overrightarrow{\mathrm{v}}_{2}\right)\right]\left[\left(\overrightarrow{\mathrm{n}} \cdot \overrightarrow{\mathrm{v}}_{1}\right)\left(\overrightarrow{\mathrm{n}} \cdot \overrightarrow{\mathrm{v}}_{2}\right)-\overrightarrow{\mathrm{v}}_{1} \cdot \overrightarrow{\mathrm{v}}_{2}\right]}{\left[1-\left(\overrightarrow{\mathrm{n}} \cdot \overrightarrow{\mathrm{v}}_{1}\right)^{2}\right]\left[1-\left(\overrightarrow{\mathrm{n}} \cdot \overrightarrow{\mathrm{v}}_{2}\right)^{2}\right]} .
\end{aligned}
$$

Here $\kappa^{2}$ and $\alpha^{2}$ mean

$$
\kappa^{2} \equiv 4 \pi \beta^{\prime} V^{-1} \sum_{R} e_{R}^{2}, \alpha^{2} \equiv 4 \pi \beta^{\prime} \sum_{R} e_{R}^{2} B\left(\beta^{\prime} m_{R}\right)
$$

with $B\left(\beta^{\prime} m_{R}\right)$ given by (27). The calculation gives for $B\left(\beta^{\prime} m\right)$

$$
B\left(\beta^{\prime} m\right)=K_{0}\left(\beta^{\prime} m\right) / V\left(\beta^{\prime} m\right)^{2} K_{2}\left(\beta^{\prime} m\right),
$$

where $K_{0}$ and $K_{2}$ are the modified Bessel functions of zeroth and second order, respectively. It can be seen that the constant $\alpha^{2}$ is positive for any positive $\beta^{\prime} m$.

From (29), the inverse Fourier transform gives the correlation function

$$
G\left(\overrightarrow{\mathrm{x}}, \overrightarrow{\mathrm{v}}_{1}, \overrightarrow{\mathrm{v}}_{2}\right)=-\frac{\beta^{\prime} e_{1} e_{2}}{r} e^{-\kappa r}+\frac{\beta^{\prime} e_{1} e_{2}}{2 \pi^{2}} \int \frac{\left(\overrightarrow{\mathrm{n}} \cdot \overrightarrow{\mathrm{v}}_{1}\right)\left(\overrightarrow{\mathrm{n}} \cdot \overrightarrow{\mathrm{v}}_{2}\right)-\overrightarrow{\mathrm{v}}_{1} \cdot \overrightarrow{\mathrm{v}}_{2}}{\left(1+\overrightarrow{\mathrm{n}}^{2} \cdot \overrightarrow{\mathrm{v}}_{1}\right)\left(1+\overrightarrow{\mathrm{n}} \cdot \overrightarrow{\mathrm{v}}_{2}\right)} \frac{e^{-i \overrightarrow{\mathrm{k}} \cdot \overrightarrow{\mathrm{x}}}}{k^{2}+\alpha^{2}} d^{3} k
$$

Here $r \equiv\left(\overrightarrow{\mathrm{x}}^{2}\right)^{1 / 2}$. We recognize in the first term of the right-hand side the well-known Debye-Hückel term of the nonrelativistic theory (except for the change $\left.\beta \rightarrow \beta^{\prime}\right)$. On the other hand, $G\left(\vec{x}, \vec{v}_{1}, \vec{v}_{2}\right)$ given by (32) satisfies the supplementary condition (11) for $t_{R}=t_{1}, \forall R$ as it must be. One can be convinced of this by noting that the integrand of (11) becomes in that case an odd function of $\vec{x}_{1}-\vec{x}_{R}$.

In (32) the integration over $k$ can be explicitly made and, alternatively, we can write

$$
G\left(\overrightarrow{\mathrm{x}}, \overrightarrow{\mathrm{v}}_{1}, \overrightarrow{\mathrm{v}}_{2}\right)=-\frac{\beta^{\prime} e_{1} e_{2}}{r} e^{-\kappa r}+\frac{\beta^{\prime} e_{1} e_{2}}{4 \pi} \int \frac{\left(\overrightarrow{\mathrm{n}} \cdot \overrightarrow{\mathrm{v}}_{1}\right)\left(\overrightarrow{\mathrm{n}} \cdot \overrightarrow{\mathrm{v}}_{2}\right)-\overrightarrow{\mathrm{v}}_{1} \cdot \overrightarrow{\mathrm{v}}_{2}}{\left(1+\overrightarrow{\mathrm{n}} \cdot \overrightarrow{\mathrm{v}}_{1}\right)\left(1+\overrightarrow{\mathrm{n}} \cdot \overrightarrow{\mathrm{v}}_{2}\right)}\left[2 \delta(\overrightarrow{\mathrm{n}} \cdot \overrightarrow{\mathrm{x}})-\alpha e^{-\alpha|\overrightarrow{\mathrm{n}} \cdot \overrightarrow{\mathrm{x}}|}\right] d \Omega_{\overrightarrow{\mathrm{n}}},
$$

where $\delta(\vec{n} \cdot \vec{x})$ is the Dirac $\delta$ and the integration is over all directions of $\vec{n}$, that is, $d \Omega_{\vec{n}}=\sin \theta d \theta d \varphi$.

Finally, for the usual two-body distribution function $f^{(2)}(1,2)=\left.F^{(2)}(1,2)\right|_{t_{1}=t_{2}}$ we get, according to (9) and (15),

$$
f^{(2)}(1,2)=F^{(1)}(1) F^{(1)}(2)\left[1-\frac{\beta^{\prime} e_{1} e_{2}}{r} e^{-\kappa r}+\frac{\beta^{\prime} e_{1} e_{2}}{2 \pi^{2}} \int \frac{\left(\overrightarrow{\mathrm{n}} \cdot \overrightarrow{\mathrm{v}}_{1}\right)\left(\overrightarrow{\mathrm{n}} \cdot \overrightarrow{\mathrm{v}}_{2}\right)-\overrightarrow{\mathrm{v}}_{1} \cdot \overrightarrow{\mathrm{v}}_{2}}{\left(1+\overrightarrow{\mathrm{n}} \cdot \overrightarrow{\mathrm{v}}_{1}\right)\left(1+\overrightarrow{\mathrm{n}} \cdot \overrightarrow{\mathrm{v}}_{2}\right)} \frac{e^{-i \overrightarrow{\mathrm{k}} \cdot \overrightarrow{\mathrm{x}}}}{k^{2}+\alpha^{2}} d^{3} k\right)
$$


with $F^{(1)}$ given by (13).

One can readily be convinced that for the $G\left(\vec{x}, \vec{v}_{1}, \vec{v}_{2}\right)$ of (32) we have

$\int G\left(\overrightarrow{\mathrm{x}}, \overrightarrow{\mathrm{v}}_{1}, \overrightarrow{\mathrm{v}}_{2}\right) d^{3} x=0$.

Hence, from (34) it becomes

$$
\int f^{(2)}(1,2) d^{3} x d^{3} u_{1} d^{3} u_{2}=V^{-1}
$$

as it must be.

\section{EXPANSION OF THE TWO-BODY CORRELATION FUNCTION: LOWEST-ORDER CORRECTION}

In this section we consider the fact $\epsilon$ is a small parameter. Then, $v^{2}(v$ standing for a typical velocity of the charges) is of order $\epsilon$. So, to begin with, let us expand the function under the integral in (33) in powers of the velocities. We obtain

$$
\begin{aligned}
G\left(\overrightarrow{\mathrm{x}}, \overrightarrow{\mathrm{v}}_{1}, \overrightarrow{\mathrm{v}}_{2}\right)= & -\beta^{\prime} e_{1} e_{2} \exp (-\kappa r) / r \\
+\left(\beta^{\prime} e_{1} e_{2} / 4 \pi\right) \sum_{p=0}^{\infty} \sum_{q=0}^{\infty} \int & {\left[\left(\overrightarrow{\mathrm{n}} \cdot \overrightarrow{\mathrm{v}}_{1}\right)\left(\overrightarrow{\mathrm{n}} \cdot \overrightarrow{\mathrm{v}}_{2}\right)-\overrightarrow{\mathrm{v}}_{1} \cdot \overrightarrow{\mathrm{v}}_{2}\right]\left(\overrightarrow{\mathrm{n}} \cdot \overrightarrow{\mathrm{v}}_{1}\right)^{p}\left(\overrightarrow{\mathrm{n}} \cdot \overrightarrow{\mathrm{v}}_{2}\right)^{q} } \\
& \times[2 \delta(\overrightarrow{\mathrm{n}} \cdot \overrightarrow{\mathrm{x}})-\alpha \exp (-\alpha|\overrightarrow{\mathrm{n}} \cdot \overrightarrow{\mathrm{x}}|)] d \Omega_{\overrightarrow{\mathrm{n}}},
\end{aligned}
$$

where $p+q$ takes only even values.

Working out the first term in this expansion we have

$$
\begin{aligned}
G\left(\overrightarrow{\mathrm{x}}, \overrightarrow{\mathrm{v}}_{1}, \overrightarrow{\mathrm{v}}_{2}\right)= & -\beta^{\prime} e_{1} e_{2} \exp (-\kappa r) / r \\
& +\left(\beta^{\prime} e_{1} e_{2} / 4 \pi\right) \int\left[\left(\overrightarrow{\mathrm{n}} \cdot \overrightarrow{\mathrm{v}}_{1}\right)\left(\overrightarrow{\mathrm{n}} \cdot \overrightarrow{\mathrm{v}}_{2}\right)-\overrightarrow{\mathrm{v}}_{1} \cdot \overrightarrow{\mathrm{v}}_{2}\right]\left[2 \delta(\overrightarrow{\mathrm{n}} \cdot \overrightarrow{\mathrm{x}})-\alpha \exp (-\alpha|\overrightarrow{\mathrm{n}} \cdot \overrightarrow{\mathrm{x}}|) d \Omega_{\overrightarrow{\mathrm{n}}}+O\left(v^{4}\right)\right.
\end{aligned}
$$

After some calculation we get explicitly

$$
\begin{aligned}
G\left(\overrightarrow{\mathrm{x}}, \overrightarrow{\mathrm{v}}_{1}, \overrightarrow{\mathrm{v}}_{2}\right)= & -\frac{\beta^{\prime} e_{1} e_{2}}{r} e^{-\kappa r} \\
& +\frac{\beta^{\prime} e_{1} e_{2}}{r} e^{-\alpha r}\left[\left(1+\frac{3}{\alpha r}+\frac{3}{\alpha^{2} r^{2}}\right] \frac{\left(\overrightarrow{\mathrm{x}} \cdot \overrightarrow{\mathrm{v}}_{1}\right)\left(\overrightarrow{\mathrm{x}}^{\prime} \cdot \overrightarrow{\mathrm{v}}_{2}\right)}{r^{2}}-\left[1+\frac{1}{\alpha r}+\frac{1}{\alpha^{2} r^{2}}\right] \overrightarrow{\mathrm{v}}_{1} \cdot \overrightarrow{\mathrm{v}}_{2}\right] \\
& +\frac{\beta^{\prime} e_{1} e_{2}}{r} \frac{1}{\alpha^{2} r^{2}}\left[\overrightarrow{\mathrm{v}}_{1} \cdot \overrightarrow{\mathrm{v}}_{2}-3 \frac{\left(\overrightarrow{\mathrm{x}} \cdot \overrightarrow{\mathrm{v}}_{1}\right)\left(\overrightarrow{\mathrm{x}} \cdot \overrightarrow{\mathrm{v}}_{2}\right)}{r^{2}}\right]+O\left(v^{4}\right) .
\end{aligned}
$$

Here, the lowest-order correction in $\epsilon$ to the classical Debye-Hückel term shows a long-range term decreasing like $1 /(\alpha r)^{2} r$ plus two sorts of shielded terms. The apparent shielded terms vary as $\exp (-\alpha r) / r$. Other relativistic shielded terms are included in the formal Debye-Hückel term $-\beta^{\prime} e_{1} e_{2} e^{\kappa r} / r$. This is so because $\beta^{\prime} \neq \beta$ in general and then $\kappa$ only coincides with the screening DebyeHückel constant for nonrelativistic plasmas. In a later paper it will be shown that $\beta^{\prime} / \beta=1+O\left(\epsilon^{2}\right)$.

In order to have a better qualitative insight on Eq. (39) let us consider the behavior of the relativistic screening constant $\alpha$ for low temperatures. Then $\beta^{\prime} m \gg 1$ and

$$
\begin{aligned}
K_{0}\left(\beta^{\prime} m\right) & \simeq K_{2}\left(\beta^{\prime} m\right) \\
& \simeq(\pi / 2 \beta m)^{1 / 2} \exp (-\beta m)
\end{aligned}
$$

which, according to Eq. (31), means

$$
B\left(\beta^{\prime} m\right) \simeq 1 / V(\beta m)^{2}, \quad \beta^{\prime} m \gg 1
$$

and so, from Eq. (30)

$$
\begin{array}{r}
\alpha^{2} \simeq 4 \pi \beta V^{-1} \sum_{R} e_{R}^{2} /\left(\beta m_{R}\right)^{2} \sim \frac{\kappa_{D}^{2}}{\left(\beta m_{e}\right)^{2}}, \\
\beta^{\prime} m_{R} \gg 1
\end{array}
$$


where $\kappa_{D}$ means the strict Debye-Hückel screening distance.

Through the relation

$$
f^{(2)}(1,2)=F^{(1)}(1) F^{(1)}(2)\left[1+G\left(\vec{x}, \vec{v}_{1}, \overrightarrow{\mathrm{v}}_{2}\right)\right]
$$

the expression of $G\left(\vec{x}, \vec{v}_{1}, \vec{v}_{2}\right)$ in (39) gives the twobody distribution function to first order in $\epsilon$. To this order, in Eq. (39), we can approximate $\beta^{\prime}$ by $\beta$, $\kappa$ by $\kappa_{D}$ and, according to (41), $\alpha$ by $\kappa_{D} /(\beta m)^{2}$.

An apparently very similar expression to (39) has been obtained before by Kosachev and Trubnikov. ${ }^{8}$ Actually, their expression agrees with ours in form but the constant $\alpha$ is very different. In fact, it can be seen that their relativistic screening distance ${ }^{9} d_{c}$ acts like a constant $d_{c} \sim\left(m V / N e^{2}\right)^{1 / 2}$ for $\beta m \gg 1$ which is to be compared with the behavior at low temperatures of our relativistic screening distance $d_{r}$, i.e., $d_{r} \sim(\beta m)^{1 / 2}$ [one obtains this relation from Eq. (41)]. Then the apparent coincidence between Eq. (39) and the results of these authors is misleading since, in fact, there is only coincidence up to or$\operatorname{der} \epsilon$.

What is the reason for these discrepancies? Actually, we do not think that the calculation of B. A. Trubnikov in Ref. 10, where he obtains our Eq. (39) with a different constant $\alpha$, is a correct calculation. In fact, Eq. (2.1) of part II of this reference is incorrect since Trubnikov takes as the one-particle distribution function the Maxwellian distribution function to the lowest order in the velocities. Obviously, since he starts his calculation from the Darwin's Lagrangian, ${ }^{11}$ which, as it is well known, retains terms up to second order in the velocities, he should have to take the Maxwellian distribution up to the same approximation. According to this, Eqs. (2.17), (2.19), (2.20), and (2.22) of Ref. 10 are wrong since in all these formulas second-order terms coming from the kinetic relativistic energy, i.e., $\frac{3}{8} \sum_{\alpha=1}^{2} m_{\alpha} v_{\alpha}^{4}$, are missing and these terms are of the same order as the first corrections to the Coulomb energy, which are retained everywhere in Trubnikov's work.

Our results do not ever coincide with those of Krizan, ${ }^{12}$ who also gets nonscreened terms for the twoparticle distribution function, because we do not find the oscillatory behavior of his distribution function. The reasons for this new disagreement are to be considered in a future paper. Also, new arguments will be given to show that the calculations referred to above from Kosachev and Trubnikov are not consistent.

In Ref. 6 the authors have found a particular solution [see Eq. (49) of that reference] to Eq. (14) modulus $O\left(\epsilon^{2}\right)$, which does not coincide with the correlation function given by (39) (even after approximating $\beta^{\prime}, \kappa$, and $\alpha$ by low temperature values as explained above). We think that this old correlation function is unphysical. On the one hand, it has no relativistic screening terms. On the other hand, the remaining relativistic long-range terms, when integrated with the Darwin Hamiltonian as to give the macroscopic energy of the plasma to order $O\left(\epsilon^{2}\right)$, give infrared divergences. This is not the case with the two-particle correlation function presented here, as will be seen in a future work. Let us remark that one can obtain the above unphysical solution by taking the limit $\alpha r \rightarrow 0$ in Eq. (39).

In the high-temperature-or ultrarelativisticlimit (which is rather academic, however, as long as pair creation is not considered), it can be seen that both $\kappa$ and $\alpha$ vanish. Then, $G\left(\overrightarrow{\mathrm{x}}, \overrightarrow{\mathrm{v}}_{1}, \overrightarrow{\mathrm{v}}_{2}\right)$ as given by Eq. (33) goes to zero with $\beta$, and the two-particle distribution function (34) becomes that of a free gas, as it must be in the ultrarelativistic limit.

Finally, the limit $r \rightarrow \infty$ of $G\left(\vec{x}, \vec{v}_{1}, \vec{v}_{2}\right)$, i.e., the long-range behavior is dominated at any temperature by a term of the form $\left(\beta^{\prime} e_{1} e_{2} / \alpha^{2} r^{3}\right)$ times a function depending only on $\overrightarrow{\mathrm{v}}_{1} \cdot \overrightarrow{\mathrm{v}}_{2}, \overrightarrow{\mathrm{x}} \cdot \overrightarrow{\mathrm{v}}_{1} / r, \overrightarrow{\mathrm{x}} \cdot \overrightarrow{\mathrm{v}}_{2} / r$. This case can be easily derived from (32) through the change $k \overrightarrow{\mathrm{r}} \equiv \overrightarrow{\mathrm{q}}$ and the neglect of $q^{2}$ compared with $\alpha^{2} / r^{2}$. That asymptotic form is clearly visible in (39) for the case of a slightly relativistic plasma.

The good behavior shown by the distribution function (34) in all the limiting cases studied in this section, and reasonable results obtained by using it (to be studied in a future paper), convince us that the function $G\left(\vec{x}, \vec{v}_{1}, \vec{v}_{2}\right)$ of Eq. (33) is the right physical solution of the integrodifferential Eq. (17).

\section{ACKNOWLEDGMENTS}

Useful discussions with $\mathrm{X}$. Barcons are gratefully recognized. Financial support for this work was provided by the Instituto de Estudios Nucleares, Madrid.
'See, for example, L. Bel, Ann. Inst. Henri Poincaré 14, 189 (1971); L. Bel and J. Martin, ibid. 22, 173 (1975) and references therein.

${ }^{2}$ L. Bel, A. Salas, and J. M. Sánchez-Ron, Phys. Rev. D I, 1099 (1973).
3J. L. Sanz, J. Math. Phys. 20, 2334 (1979).

${ }^{4}$ R. Lapiedra and A. Molina, J. Math. Phys. 20, 1308 (1979); R. Lapiedra, F. Marqués, and A. Molina ibid. 20, 1316 (1979).

${ }^{5}$ R. Blanco, L. Pesquera, and E. Santos, Phys. Rev. D (in 
press).

${ }^{6}$ R. Lapiedra and E. Santos, Phys. Rev. D $\underline{23}, 2181$ (1981).

${ }^{7}$ N. G. Van Kampen, in Fundamental Problems in Statistical Mechanics, edited by E. G. D. Cohen (NorthHolland, Amsterdam, 1968).

${ }^{8}$ V. V. Kosachev and B. A. Trubnikov, Nucl. Fusion 9 , 53 (1969).
${ }^{9}$ B. A. Trubnikov, Nucl. Fusion $\underline{8}, 51$ (1968); $\underline{8}, 59$ (1968).

${ }^{10}$ B. A. Trubnikov and V. V. Kosachev, Zh. Eksp. Teor. Fiz. 54, 940 (1968) [Sov. Phys._JETP 27, 501 (1968)].

${ }^{11}$ See, for example, L. Landau and E. Lifchitz, Théorie des Champs (Editions Mir, Moscow, 1970). The original reference is C. G. Darwin, Philos. Mag. 39, 537 (1920).

12J. E. Krizan, Phys. Rev. A $\underline{10}, 298$ (1974). 